\title{
On Reactive Force in Thin Unclosed Conductor and Displacement Current
}

\author{
Sergey A. Gerasimov
}

\section{ABSTRACT}

\begin{abstract}
The linear momentum density carried by electromagnetic fields creates the hidden force acting on the displacement current between ends of an unclosed conductor with alternative electric current. This force compensates the self-force exerted by the unclosed conductor with zero thin. The magnetic field produced by displacement current does not contribute to the force acting on the conductor. The unclosed conductor can move under action of the self-force.
\end{abstract}

At small heights of cylindrical open conductor, the reactive force equivalent to the self-force becomes very large.

Keywords: Abraham's force, alternative current, conservation of momentum, displacement current, hidden momentum, self-force.
Published Online: September 04, 2021

ISSN: $2684-4451$

DOI :10.24018/ejphysics.2021.3.5.100

\section{S. A. Gerasimov*}

Department of General Physics, Faculty of Physics, Southern Federal University, Russian Federation.

(e-mail: gsim1953@mail.ru)

*Corresponding Author

\section{INTRODUCTION}

An alternating electric current, unlike a direct one, can flow in an unclosed conductor. The closure of the total electric current is provided by the so-called displacement current [1]. On the other hand, an unclosed conductor with a current exerts the so-called self-force, that is, the force with which the conductor acts on itself [2]. For direct current, the self-force is experimentally discovered [3] and fully corresponds to the law of conservation of momentum, according to which the sum of all forces acting in a closed system is strictly equal to zero [4]. In other words, the total force acting on one open part of the conductor is compensated by an equal in magnitude and opposite direction force acting on the rest of the closed electrical circuit.

Two questions arise that have not yet been answered. First, does the displacement current act on the conduction current flowing in an open conductor? Since the displacement current is proportional to the change in the induction of the electric field with time, and the alternating electric field generates an alternating magnetic field, then the ampere force must act on the conduction current. The second question seems rather strange. Whether the magnetic field created by an open conductor acts on the displacement current, creating something like an Ampere force? The fact is that it is not fields that interact, but currents and charges.

An attempt to answer these questions is quite justified. First, there is an indirect experimental confirmation of the action of an open conductor with a current on the displacement current, however, referring not to force, but to the torque [5]. Secondly, if an open conductor with direct or alternating current acts on itself, then there must be the force acting on the field. Since the electromagnetic field has not only momentum but also mass, the transfer of momentum to the field is a completely real process that can be described theoretically. It is hard enough to dwell on the consideration of an absolutely thin open conductor with an alternating electric current. Of greatest interest, including from a practical point of view, is an attempt to answer these two questions as applied to a real conductor, say, which is a part of a thin metal cylinder.

\section{REACTION FORCE AND ABRAHAM's FORCE}

In the physics of electromagnetism, the Abraham force is the so-called the radiation reaction force [6], [7]. The reaction force is the force with which the support acts on the body in question. In this case, a support means an alternating electromagnetic field described by a displacement current. The displacement current creates a magnetic field that must act on the conductor, exerting a force that is commonly called the Abraham force.

It is convenient to consider a part of a cylindrical conductor of radius $R$ and height $H$ in which an alternative electric current of density $\mathbf{j}$ flows (Fig. 1).

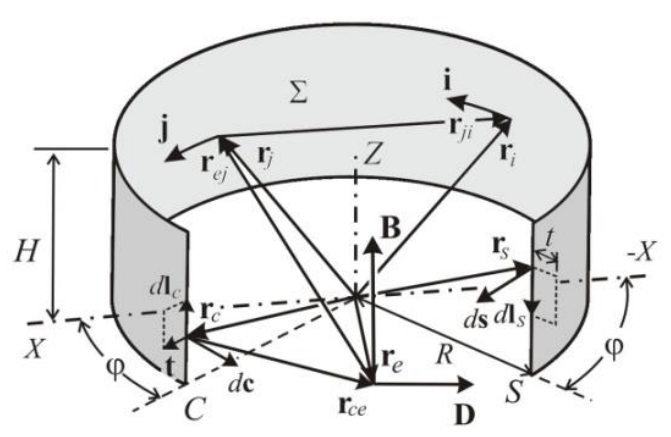

Fig. 1. A part of a cylinder with alternative electric current. 
A line electric charge with the linear charge density $\lambda\left(\mathbf{r}_{c}\right)$ at a point with radius-vector $\mathbf{r}_{c}$ produces the electric field inductance:

$$
d \mathbf{D}_{c}=\frac{1}{4 \pi} \frac{\lambda\left(\mathbf{r}_{c}\right) d l_{c} \mathbf{r}_{c e}}{r_{c e}^{3}}
$$

where $j\left(\mathbf{r}_{c}\right)=d \lambda\left(\mathbf{r}_{\mathrm{c}}\right) / d t$ is the conductive current in the conductor at point with radius-vector $\mathbf{r}_{c}$. Since

$$
\left[\nabla \times \frac{\left[\mathbf{j}\left(\mathbf{r}_{c}\right) \times \mathbf{r}_{e j}\right]}{r_{e j}^{3}}\right]=-\nabla \frac{\left(\mathbf{j}\left(\mathbf{r}_{c}\right) \mathbf{r}_{e j}\right)}{r_{e j}^{3}}
$$

that the magnetic field produced by the displacement current may be described by the expression equivalent to the BiotSavart force law. This means that this magnetic field exerts the force on the part of a cylinder:

$$
\mathbf{F}_{r c}=\frac{\mu_{0}}{(4 \pi)^{2}} \int_{\Sigma}\left[\mathbf{j}\left(\mathbf{r}_{j}\right) \times \int_{V} d^{3} r_{e} \frac{\left(\mathbf{j}\left(\mathbf{r}_{c}\right) d \mathbf{l}_{c}\right)\left[\mathbf{r}_{c e} \times \mathbf{r}_{e j}\right]}{r_{c e}^{3} r_{e j}^{3}}\right] d \sigma
$$

Integral over volume $V$ occupied by the displacement currents can be rewritten using a vector identity:

$$
\left[\left(\nabla_{e} \frac{1}{r_{e j}}\right) \times\left(\nabla_{e} \frac{1}{r_{c e}}\right)\right]=-\left[\nabla_{e} \times \frac{1}{r_{c e}}\left(\nabla_{e} \frac{1}{r_{e j}}\right)\right]
$$

that enables to transform this integral in the surface integral:

$$
\int_{V} d^{3} r_{e}\left[\nabla \times \frac{1}{r_{c e}}\left(\nabla \frac{1}{r_{e j}}\right)\right]=\oint_{S}\left[d \mathbf{s} \times \frac{1}{r_{c e}}\left(\nabla \frac{1}{r_{e j}}\right)\right]
$$

over area $S$ of infinite radius. The functional structure of the integrand guarantees the vanishing of the force due to the rectilinear charged part $C$ of the cylindrical conductor: $\mathbf{F}_{r c}=0$. The same relates to the force due to the part $S$. This means that no reaction magnetic force acts on the conductor:

$$
\mathbf{F}_{r}=\mathbf{F}_{r c}+\mathbf{F}_{r s}=0
$$

This was due to the spherical symmetry of the displacement currents. The only approximation used is the Coulomb character of the electric field induction (1) created by the elements of the edges $C$ and $S$. Whether this is correct or not, it can be seen from the subsequent calculations. The full linear momentum of the system, including the momentum of the electromagnetic field, must be conserved. In other words, the total force acting on the system must be zero.

\section{HIDDEN FORCE}

The linear momentum density carried by electromagnetic fields is related to the Poynting vector [8]:

$$
\mathbf{p}_{e m}=[\mathbf{D} \times \mathbf{B}]
$$

where $\mathbf{D}$ is the net electric field inductance produced by the displacement current of whole the system. Neglecting the contribution of the magnetic field created by the displacement currents, one can assume that the force attributed to the field due to the edge $C$ is:

$$
\mathbf{F}_{a c}=\frac{\mu_{0}}{(4 \pi)^{2}} \int_{C}\left[\mathbf{j}\left(\mathbf{r}_{c}\right) d l_{c} \int_{\Sigma} d \sigma \int_{V}\left[\frac{\mathbf{r}_{c e}}{r_{c e}^{3}} \times \frac{\left[\mathbf{j}\left(\mathbf{r}_{j}\right) \times\left(-\mathbf{r}_{j e}\right)\right]}{r_{j e}^{3}}\right] d^{3} r_{e}\right.
$$

Introducing the vector potential

$$
\mathbf{B}\left(\mathbf{r}_{e}\right)=\left[\nabla \times \mathbf{A}\left(\mathbf{r}_{e}\right)\right]
$$

and taking into account that

$$
\nabla_{e} \frac{1}{r_{c e}}=-\frac{\mathbf{r}_{c e}}{r_{c e}^{3}}
$$

the integrand in (5) can be transformed by the following manner:

$$
\left[\left(\nabla_{e} \frac{1}{r_{c e}}\right) \times\left[\nabla \times \mathbf{A}\left(\mathbf{r}_{e}\right)\right]\right]=\left[\nabla \times \frac{\mathbf{A}\left(\mathbf{r}_{e}\right)}{r_{c e}}\right]+\frac{1}{r_{c e}} \Delta \mathbf{A}\left(\mathbf{r}_{e}\right)
$$

Here, the first volume integral of (6) can be converted in surface integral of infinite radius using the rotor theorem:

$$
\int_{V}\left[\nabla \times \frac{1}{r_{c e}} A\left(r_{e}\right)\right] d^{3} r_{e}=\oint_{S}\left[d \mathbf{s} \times \frac{1}{r_{c e} r_{j e}} \mathbf{j}\left(\mathbf{r}_{j}\right)\right] \rightarrow
$$

and since the surface element $d \mathbf{s}$ is collinear to $\mathbf{r}_{c e}$ that this integral is zero

$$
\rightarrow-\left[\mathbf{j}\left(\mathbf{r}_{c}\right) \times \oint_{S} \frac{\mathbf{r}_{c e}}{r_{c e}^{3}} d s\right]=0
$$

The second integral of (6) is easily calculated using a property

$$
\Delta \frac{1}{r_{j e}}=-4 \pi \delta\left|\mathbf{r}_{e}-\mathbf{r}_{j}\right|
$$

and finally, the hidden force attributed to the field is:

$\mathbf{F}_{a}=\frac{\mu_{0}}{4 \pi}\left(\int_{C} j\left(\mathbf{r}_{c}\right) d l_{c} \frac{\mathbf{j}\left(\mathbf{r}_{j}\right)}{\left|\mathbf{r}_{j}-\mathbf{r}_{c}\right|} d \sigma-\int_{S} j\left(\mathbf{r}_{s}\right) d l_{s} \int_{\Sigma} \frac{\mathbf{j}\left(\mathbf{r}_{j}\right)}{\left|\mathbf{r}_{j}-\mathbf{r}_{s}\right|} d \sigma\right)$

The density of this force is equal to the time derivative of the hidden linear momentum. Formally speaking, this force is absolutely equivalent to a force exerted by displacement current elements due to magnetic field created the 
conductive current flowing in a conductor. Something is lost since

$$
\mathbf{F}_{r}+\mathbf{F}_{a} \neq 0
$$

\section{SELF-FORCE}

There is not enough impact on the conductor. The only body that can create the missing effect is the body itself. This impact can be described by force [3], [4]:

$$
\mathbf{F}_{s}=\frac{\mu_{0}}{4 \pi} \int_{\Sigma} d \sigma \int_{\Sigma}\left[\mathbf{i}\left(\mathbf{r}_{i}\right) \times \frac{\left[\mathbf{j}\left(\mathbf{r}_{j}\right) \times \mathbf{r}_{j i}\right]}{r_{j i}^{3}} d \sigma\right.
$$

where the surface current elements $\mathbf{i} d \sigma$ and $\mathbf{j} d \sigma$ belong to the same part of the cylinder shown in Fig. 1.

In the vector identity

$$
\left[\mathbf{i} \times\left[\mathbf{j} \times \mathbf{r}_{j i}\right]\right]=\mathbf{j}\left[\mathbf{i} \mathbf{r}_{j i}\right]-\mathbf{r}_{j i}[\mathbf{j i}]
$$

the second term is anti-symmetric with respect to the replacement of current elements and does not contribute to the Biot-Savart force (9).

Since

$$
\left.\frac{\mathbf{r}_{i}-\mathbf{r}_{j}}{r_{j i}^{3}}\right]=-\nabla_{i} \frac{1}{r_{j i}}
$$

and

$$
(\mathbf{J} \nabla \rho)=(\nabla(\rho \mathbf{J})-\rho(\nabla \mathbf{J})
$$

then the integral (9) can be rewritten as:

$$
\mathbf{F}_{s}=-\frac{\mu_{0}}{4 \pi} \int_{\Sigma} \mathbf{j}\left(\mathbf{r}_{j}\right) d \sigma \int_{\Sigma}\left(\nabla \frac{\mathbf{i}\left(\mathbf{r}_{i}\right)}{r_{j i}}\right) d \sigma
$$

There is no theorem that would allow us to transform the surface integral into an integral over a contour. Therefore, it is better to make this integral volume, assuming that the circuit with currents has a thickness $\delta$. Multiplying and dividing the inner integral (9) by $\delta$, and using the divergence theorem, one can write:

$$
\mathbf{F}_{s}=-\frac{\mu_{0}}{4 \pi} \int_{\Sigma} \mathbf{j}\left(\mathbf{r}_{j}\right) d \sigma \frac{1}{\delta} \oint \frac{\left(\mathbf{i}\left(\mathbf{r}_{i}\right) d \Sigma\right)}{r_{j i}}
$$

where $d \Sigma$ is the element of the surface of the "thickened" conductor. Electric current flows only through the ends of the conductor, for which

$$
d \Sigma_{c}=d \mathbf{c}=\left[d \mathbf{l}_{c} \times \delta\right] ; d \Sigma_{s}=d \mathbf{s}=\left[d \mathbf{l}_{s} \times \delta\right]
$$

Keeping in mind that vectors $d \Sigma, d \mathbf{l}$. and $\delta$ are mutually orthogonal and getting rid of the mixed product, one obtains

$$
\mathbf{F}_{s}=-\frac{\mu_{0}}{4 \pi}\left(\int_{C} j\left(\mathbf{r}_{c}\right) d l_{c} \int_{\Sigma} \frac{\mathbf{j}\left(\mathbf{r}_{j}\right)}{r_{j c}} d \sigma-\int_{S} j\left(\mathbf{r}_{s}\right) d l_{s} \int_{\Sigma} \frac{j\left(r_{j}\right)}{r_{j s}} d \sigma\right)
$$

where the replacement $i \rightarrow j$ is naturally made.

The result is quite interesting. The force with which the unclosed conduction currents act on the field (7) is equal in magnitude and opposite in direction to the force with which the conduction current in the conductor acts on itself (12):

$$
\mathbf{F}_{r}+\mathbf{F}_{a}+\mathbf{F}_{s}=0
$$

This means that the total linear momentum conserves.

\section{SINGULARITY}

Everything that has been said above is of practical interest if the self-force has a noticeable magnitude. On the other hand, the calculation results are valid for any conductor with zero thickness. An example is needed to assess the force of self-action, which plays the role of reactive force.

For cylindrical surface, the position of the current element $\mathbf{j} d \sigma$ can be written by radius-vector:

$$
\mathbf{r}_{j}=-R \cos \vartheta_{j} \mathbf{e}_{x}-R \sin \vartheta_{i} \mathbf{e}_{y}+h \mathbf{e}_{z}
$$

Hence

$$
d \mathbf{r}_{j}=R \sin \vartheta_{j} \mathbf{e}_{x}-R \cos \vartheta_{i} \mathbf{e}_{y}
$$

The same is for current elements $\mathbf{i} d \sigma$. The only distinguished direction of the force is the axis $Y$, therefore

$$
\left.d \mathbf{r}_{j}\left(d \mathbf{r}_{i} \mathbf{r}_{j i}\right)\right|_{y}=-R^{3} \sin \left(\vartheta_{j}-\vartheta_{j}\right) \cos \vartheta_{j} d \vartheta_{j} d \vartheta_{i}
$$

and

$$
r_{j i}\left(\vartheta_{i}, \vartheta_{j}, h_{i}, h_{j}\right)=\left(4 R^{2} \sin ^{2}\left(\left(\vartheta_{j}-\vartheta_{j}\right) / 2\right)+\left(h_{i}-h_{j}\right)\right)^{1 / 2}
$$

and finally, the $Y$-component of the self-force (9) is:

$$
F_{s}=\frac{\mu_{0} I^{2} R^{3}}{4 \pi H^{2}} \int_{-\phi}^{\pi+\phi} d \vartheta_{j} \int_{-\phi}^{\pi+\phi} d \vartheta_{i} \int_{0}^{H} d h_{j} \int_{0}^{H} \frac{\sin \left(\vartheta_{i}-\vartheta_{j}\right) \cos \vartheta_{j}}{r_{j i}^{3}\left(\vartheta_{i}, \vartheta_{j}, h_{i}, h_{j}\right)} d h_{i}(14)
$$

where $j=I / H$.

Only three integrals in (14) can be calculated analytically. Note that the reduced value $F_{s} / I^{2}$ of the self-force (14) depends only on $H / R$ and the angle $\varphi$. There is no need, therefore, to write down the cumbersome transformation result of the integral (14). The goal is to make sure that this force can be very large. This is shown in Fig. 2. 


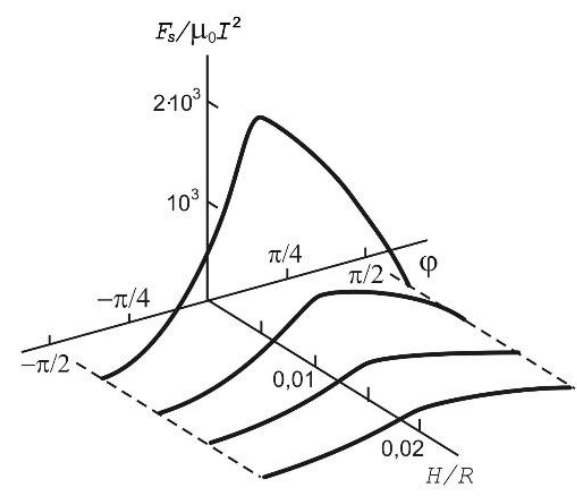

Fig. 2. Singularity of the self-force at $H \rightarrow 0$.

At low heights of a cylindrical conductor, the self-force is singular, that is, at $H \rightarrow 0$ it becomes infinite. The divergence of the self-force [9], therefore, is a physical and real phenomenon. This is primarily due to the infinite conduction current density $j$ at a given current strength $I$ and a given conductor height $H$. At any heights of the conductor, the force is maximum at $\varphi=0$.

\section{CONCLUSION}

The hidden force transmitted by an open conductor to an alternating electromagnetic field compensates for the selfforce. This is the main result of this work. Such compensation is absolute. Only this result satisfies the momentum conservation law. The question remains: how to create an alternating electric current in an open conductor? This can be done using Foucault currents [10]. Foucault currents are the alternative electric currents induced in masses of conducting metal that is immersed in a variable magnetic field. These currents are no different from ordinary conduction currents, therefore they may be unclosed.

\section{REFERENCES}

[1] P. A. Mallo, "A remark on Maxwell's displacement current," American Journal of Physics, vol. 40, pp. 110-113, July 1972.

[2] S. A. Gerasimov, "Self-interaction and vector potential in magnetostatics," Physica Scripta, vol. 56, no. 5, pp. 462-464, 2014.

[3] G. Cavalleri, G. Bettoni, E. Tonni, G. Spavieri, "Experimental proof of standard electrodynamics of measuring the self-force on a part of current loop," Physical Review E., vol. 58, no. 2, pp. 2505-2517, 1998.

[4] S. A. Gerasimov, "Interaction of current elements, conservation of momentum and pseudo-self-action in magnetostatics," Applied Physics and Mathematics, vol. 2, no. 1, pp. 3-6, 2014.

[5] G. M. Graham, D. G. Lahoz, "Observation of static electromagnetic momentum in vacuo," Nature, vol. 285(5761), pp. 154-155, 1980.

[6] M. Abraham, Classical Theory of Electricity and Magnetism, London: Blackie \& Son, 1948.

[7] F.Rohrlich, "The self-force and radiation reaction," American Journal of Physics, vol. 68, no. 12, pp. 1109-1112, 2000.

[8] D. Babson, S. P. Reynolds, "Hidden momentum, field momentum and electromagnetic impulse," American Journal of Physics, vol. 77, no. 9, pp. 826-833, 2009.

[9] N. Graneau, "The finite size of the metallic current element," Physics Letters A, vol. 147, no. 2-3, pp. 92-96, 1990.

[10] R. K. Wngsness, Electromagnetic Fields, New-York: John Wiley, 1986.

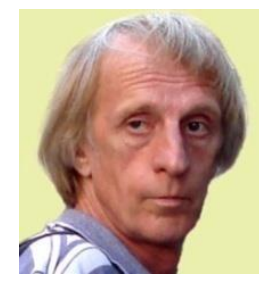

Sergey A. Gerasimov is an Associate Professor of Faculty of Physics of Southern Federal University. His research interests include Mechanics and Electrodynamics. He received a $\mathrm{PhD}$ degree in Physics and Mathematics from Rostov State University, Rostov-on-Don, Russia. He has published about 200 scientific papers in International and Russian journals. $\mathrm{He}$ has experience in the academic field lecturing for theoretical and experimental courses. 